\title{
The Voynich Manuscript Interprupted as Tibetan
}

\author{
Paul TE Cusack* \\ BScE, Dule, Saint John, Canada
}

Submission: June 18, 2018; Published: July 20, 2018

*Corresponding author: Paul TE Cusack, BScE, Dule, 1641 Sand y Point Rd, Saint John, NB, Canada E2K 5E8, Tel: (506) 214-3313;

Email: St-Michael@hotmail.com

\begin{abstract}
The Voynich Manuscript is written in Tibetan. The Hebrew tribe of Manasseh likely passed through Tibet on their way to North America. The left behind the script that was detained by the Tibetan Monks. This is why the American Indian Language reflects the mysterious language. It is also why it appears to be Semitic. Abraham was Semitic. The paper presents a table that will allow the interested reader to translate the entire document. It could more easily be read simply by someone who reads Tibetan handwriting.
\end{abstract}

Keywords: Vaaaaoynich Manuscript; Tibetan Language; American Indian; Manasseh; Israel; Script; Tablets; Handwriting; Mother Nature; Microbiology; Astrology; Astronomy; Anatomy; Biology; Polymer; Scriptures; Letters; Alphabet

\section{Introduction}

On December 10, 2014, I was looking for a new unsolved problem. I came across a bright webpage that list 25 of the most intriguing Mysteries of the present time. I spotted the Voynich Manuscript riddle, which I had not heard of before hitherto. I thought to myself, that is the next problem for me. I had already decoded the Minoan Tablets. It was exciting. So, I gave the Voynich Manuscripts a try.

At first glance, the print looked to be German to me on the internet site "Voynich Manuscript Images." [1]. Then I realized that all the illustrations pointed to Biology, Anatomy, Microbiology, Astrology, perhaps Astronomy, and Greek goddesses. The whole thing spoke to me as a work on Mother Nature. I thought perhaps it was written by a woman, a Lesbian (Lesbo), or a witch. I realized that there were sometimes 7 women or 8. I looked up the Greek goddesses. There are 7 of them. That was a clue. Then I realized that one of the diagrams (page 74) had three concentric circles. I knew that the Hebrew Alphabet could be converted to the alphanumeric. So, could the Greek. When I counted the number of figures in that diagram, I counted 2 Fish, 10 and 19. I thought of prime numbers. I knew from my Mathematical Physics that Egypt had encoded in their pyramids a lot of complex Mathematics, including calculus. I knew that Hebrew people learned this mathematics from Moses and Joseph when they sojourned into Egypt as we know from the Hebrew Scriptures. From my previous research knew that the Hebrews took this cosmic information everywhere they went including the UK (Stone Henge) and Ireland (St Columba's Psalter) and as far as the Americas as the Mayans in the Tribe of Manasseh (twin son to Ephraim of Joseph the patriarch). As any good Mormon at your door will tell you the Native North American peoples descend from Manasseh. So, if Manessah went East, they crossed India and china likely. They are Haplogroup "D" which is Chinese or Native North Americans. (I have that DNA too).

Of course, Tibet (Tibit) is between India and China in the Himalayan Mountains. The area is most famous for political unrest with china and its Tibetan Buddhist Monks. The manuscript figure I focussed on first was the one that translated to BIT or TIB. I realized going inward it is TIB, and outward BIT. That spells TIBIT or Tibet. I looked up the Tibetan "alphabet" online. After studying it for about 10 minutes, I figured that alphabet contains the cursive handwriting found in the manuscript. I found an online Tibetan to English translator Tool which proved extremely important in deciphering the manuscript.

It takes imagination to see how the cursive handwriting relates to the formal "letters" (They are actually not letter per say; they are symbols dor words in a primitive language. It is perhaps advanced beyond the Hebrew Ten commandments symbolism I uncovered, but not as advanced as the Hebrew Alphabet. Moses of course was given the Ten Commandments c.1200 B. C.E. The tribe of Manasseh had a parcel of land among the tribes of Israel. When or why they left, I do not know.

I also knew from examination of the illustrations that whomever wrote the manuscript had access to a microscope and human cadavors. The Chinese are known to have had the 
microscope since c 2000 B.C.E. They could see small bacteria like creatures. They deciphered DNA in stringy semen and knew there was a code in that DNA. The scripter also knew of Evolution from Octopus to Horse. Of course, many plants were illustrated. In addition to all the biology, there was the Astrology. The Sun was illustrated, as well as the 4 Seasons. The cosmos was illustrated as a circle with many stars of no pattern that I could discern except for the numerology. I don't know anything about Astrology nor Astronomy except Cosmology. Still the message is clear in the manuscript: it was about Nature- Mother Nature judging from the Nymphs who appear pregnant.

My purpose is not to detail these illustrations; it is to attempt to decipher the mysterious script [2]. Once I knew that TIBIT was the key, I just had to translate the cursive to the formal Tibetan Alphabet chart also available on line under Images. It became clear when I could read the script that the Tibetan Language was the key and the book was likely the Tibetan Monks "Bible". If Roger Bacon wrote it, then he knew Tibertan language well enough to write a book in it. When I uncovered on Wikipedia that the Qing Dynasty fell in Tibet in 1912, the year Voynich bought the script in Italy, the picture was becoming clear. Someone had smuggled out a copy of the Tibetan Book of Knowledge. What they call it I do not know.

As I translated the script letter by letter, I could see that first, the text did not directly refer to the illustrations; and second that the letters are words much like the Chinese script. Letter became words, and words became sentences which made sense for a "Tibetan Bible". The only proof I have is in the fact that the script can be read. I don't have advanced linguistic training and tools to offer. Things such as radio carbon dating, chemical analysis of the pigments, etc could rule out my theory. As it stands, I suggest the manuscript is a Tibetan Bible written by someone who knew how to write their language. I suspect, it was an unknown Tibetan Monk who wrote the manuscript in the 15 Century. I can't see why Western Scientist oger Bacon would write such a primitive script, although one hidden from the Churchmen of the day.

What I present here is the Tibetan translation of a few words that give a feel for the manuscript. I figured out 19 out of 42 characters or about $45 \%$ of them. I don't intend to translate the entire book as perhaps any Tibetan Monk could read it handily. Admittedly, there is the odd character I can't explain. Perhaps a Tibetan Monk can. Thanks to Prof Shell of Harvard for his useful comments. The theory, right or wrong is entirely mine (Figure 1). My guess is that the Vioynic Manuscript (Mother Nature's Manusrcipt) was written in German (False) perhaps by a woman (FALSE)? The alphabet used is Greek and Latin because that is where the Nymph myths come from Greek Mythology. It is about the Universe as she projected it. There are illustrations on the Sun, (24 hours of the day), Cosmology (Astrology or Astronomy), Mathematics (Prime numbers), the cell (they had a microscope), Anatomy (Aorta, Myan Sheath) microbiology, DNA (the coding and the polymer), Botany, Evolution (octopus and the elephant). Its a book on the universe, and life. Its about "Mother Nature" as a religion. There must have been 7 famous nymphs plus the one who wrote the manuscript totaling 8 .

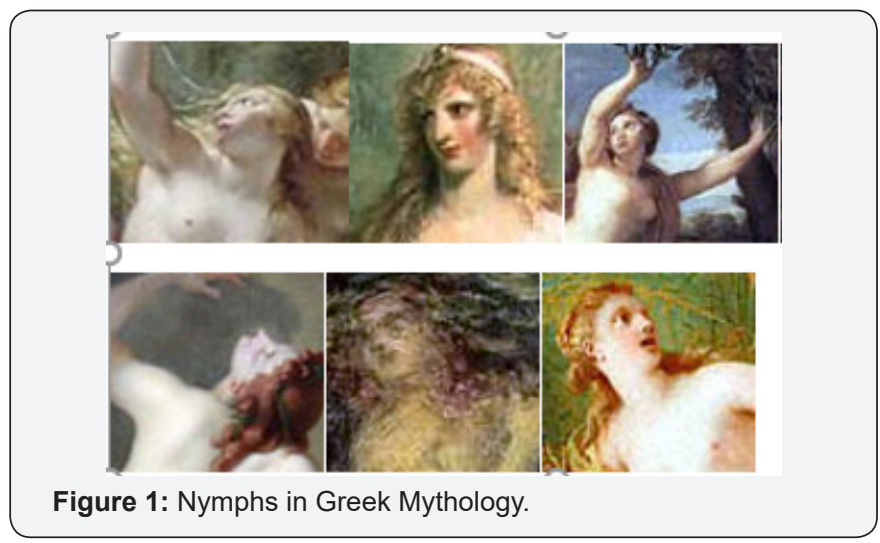

My guess is that Homer is a big clue. You'll find these 7 names below in the text I suspect. That will decode your alphabet (Figure 2). So there are 2 fishes (B), 10 animals (I), and 19 women (T). That decodes from the Greek alpha numeric to "BIT" or "TIB". Is that Mother Nature's Son? (TIBET) Beatles: It was inspired by a lecture given by the Maharishi Mahesh Yogi while the Beatles were in India The Quing dynasty collapsed in 1912 the year the Polish Votnich purchased the manuscript. Prime Numbers $=2,3,5,7,11,17,192+10+400=412$ OR: $2+20+400=422=\mathrm{Pi}-\mathrm{e}=0.42331 / 412=0.588 \quad 1 / 0.588=1.7$ (Chemistry Chemistry) $=$ delta $=\mathrm{d}$ TIBIZ=TIBET This Manuscript is a Tibetan Monk's Book on Mother Nature.

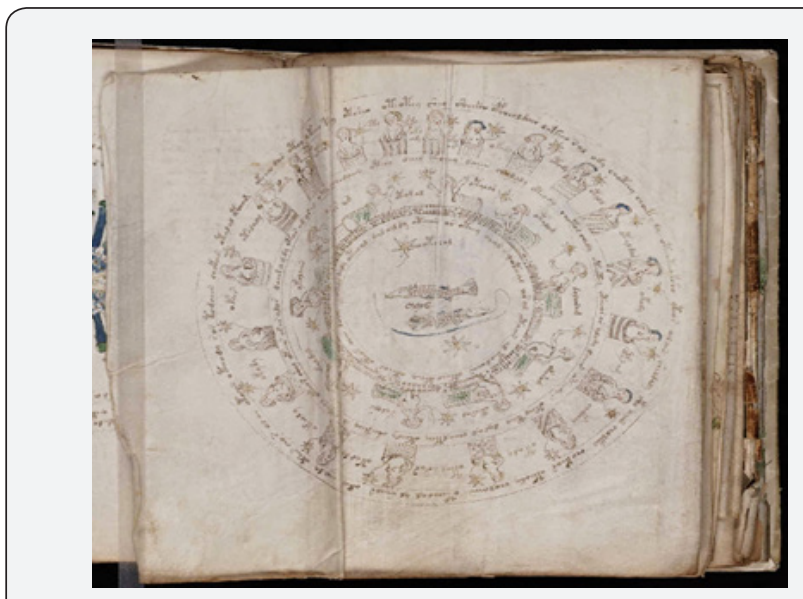

Figure 2:That decodes from the Greek alpha numeric to "BIT" or "TIB".

TIBIT $=2^{\wedge} 40=1.099$ Trillion

IO $10+70=71$

$1 / 71=0.14081 / 0.1408=859$

ECHO $5+20+8+70=103$

8572=HEOC(?) (Figures 3-7)

I'll never forget when I went to an interview at Irving Oil, the largest employer in the city. The mature 45 -year-old woman 
said to me, I started at the bottom in a call center with a degree in Psychology, why shouldn't you? It was for an Engineering/ MBA position. Then she said, you've proven you can learn from a book. What else can youuuuu doooooo? So, then I went to an interview at Ocean Steel, another Irving company. The 22-yearold girl asked me why I wasn't a member of the Association (of Professional Engineers)? I said on page 1 of my 2-page resume, on the first line is my name in big black letters with P.Eng. after my name. That means I'm a member of the Association. Oh that's what that means. Then on page 2 I said, it states at the bottom of the 2-page resume Memberships: Association of Professional Engineers. Good thing she wasn't hiring doctors. What does Dr stand for anyway? (Table 1) There has got to be a Newfoundlander joke in there somewhere! (Figures 8 \& 9) DM isa the goddess Demeter.

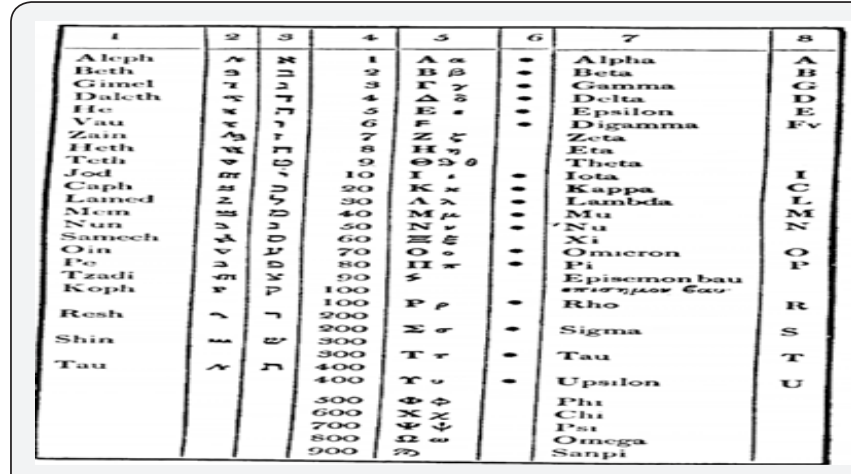

Figure 3: Tibetan Monk's Book on Mother Nature.

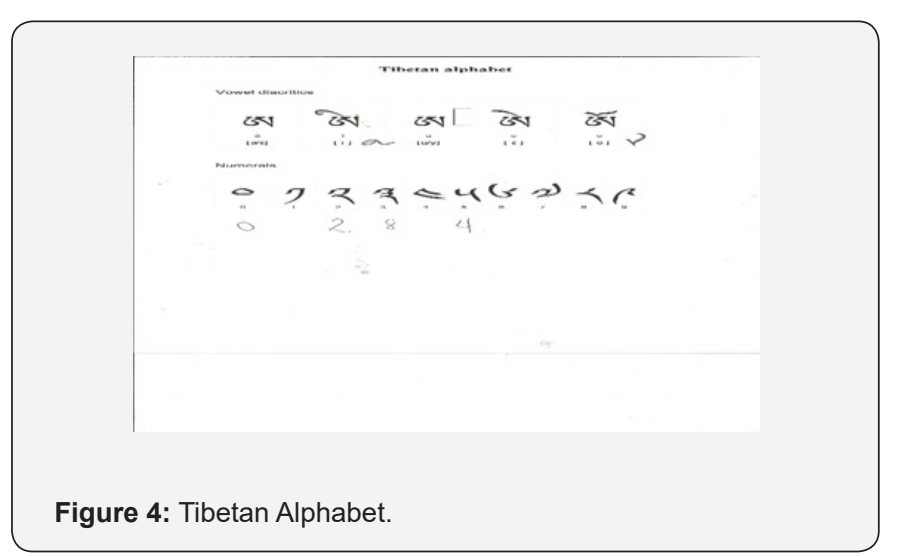

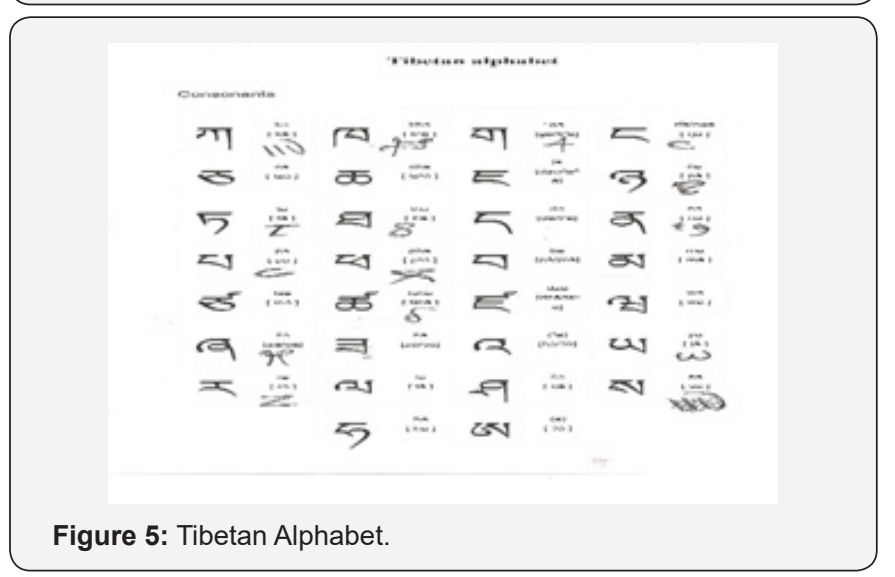

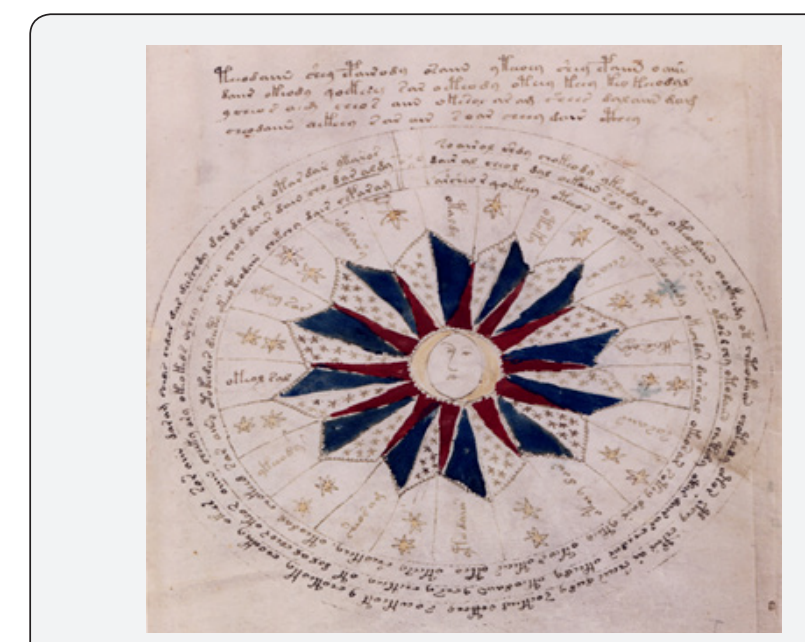

Figure 6: Bottom in a call center with a degree in Psychology.

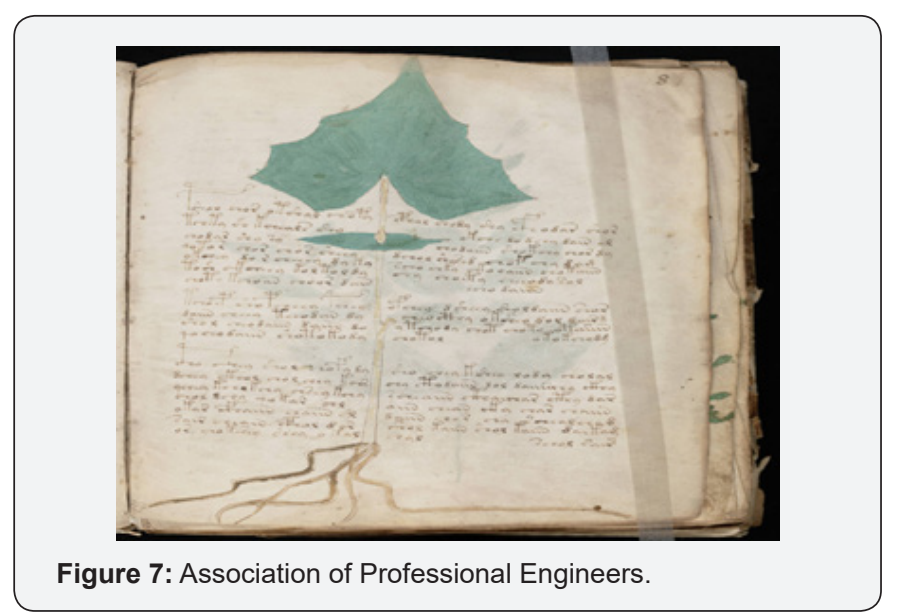

\section{saryo $\langle 49$ \\ THA I O I O $8 \mathrm{Za} \mathrm{Ha}$}

Figure 8: All Goddesses, A Kiss Dm In Ecstasy.

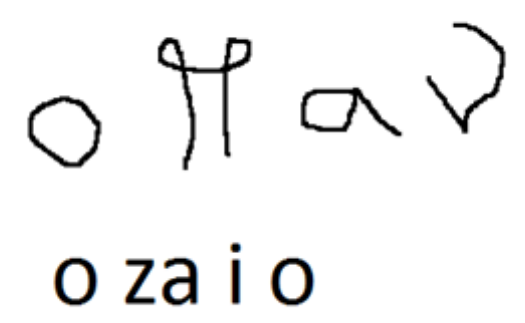

Figure 9: kiss goddess dm, the invisible entity. 


\section{Global Journal of Archaeology \& Anthropology}

Table 1: All Goddesses, A Kiss Dm In Ecstasy.

\begin{tabular}{|c|c|c|}
\hline \multirow{4}{*}{8} & OT & $\begin{array}{l}\text { [1132] bod yig gsal byed bcu pa 'di'i nga ro 'don tshul la skye gnas so dang/ byed pa lce rtse/ nang gi rtsol ba mgrin pa } \\
\text { phye ba dang/ phyi'i rtsol ba srog chen sgra med/ ming gzhi'i ma ning gi yi ge zhig ... }\end{array}$ \\
\hline & $\mathrm{JV}$ & $\begin{array}{c}\text { everything, all, total, foolishness, mental darkness, entrance to cognition of all things, instability of all things, number 10, } \\
\text { inferior-quality }\end{array}$ \\
\hline & IW & $\begin{array}{l}\text { THA * everything, all, total. THA [bod yig gsal byed bcu pa 'di'i nga ro 'don tshul la skye gnas so dang, byed pa lce rtze, nang } \\
\text { gi rtzol ba mgrin pa phye ba dang, phyi'i rtzol ba srog chen sgra med, ming gzhi'i ma ning gi yi ge zhig] everything, all, total }\end{array}$ \\
\hline & RY & tha everything, all, total \\
\hline \multirow{2}{*}{ खें } & JV,IW,RY & beer \\
\hline & $\mathrm{JV}$ & *, lynx, invisible entity \\
\hline 柿 & IW, RY & a kiss \\
\hline\langle & & [not found] \\
\hline \multirow{6}{*}{$\exists \cdot$} & JH-ENG & food; to eat \\
\hline & JH-T & bza'/za/bzas/zos/ \\
\hline & OT & $\begin{array}{l}\text { [2441] bod yig gsal byed nyer gnyis pa 'di'i nga ro 'don tshul la skye gnas so dang/ byed pa lce rtse/ nang gi rtsol ba mgrin } \\
\text { pa phye ba dang/ phyi'i rtsol ba srog chen dro zhing sgra med pa/ ming gzhi'i mo yig cig ... }\end{array}$ \\
\hline & DM & $\begin{array}{l}\text { as a part of women's names, immediately following the clan name, may also be spelled bza', or, more problematically, tsha } \\
\text { or btsa'. }\end{array}$ \\
\hline & IW & $\begin{array}{l}\text { ZA [bod yig gsal byed nyer gnyis pa 'di'i nga ro 'don tshul la skye gnas so dang, byed pa lce rtse, nang gi rtsol ba mgrin } \\
\text { pa phye ba dang, phyi'i rtsol ba srog chen dro zhing sgra med pa, ming gzhi'i mo yig cig ] \{dbang-gsum-three profound } \\
\text { empowerments [Gd-mk]. ZA [R] }\end{array}$ \\
\hline & $\mathrm{RY}$ & accept, take! \\
\hline \multirow{4}{*}{5} & OT & $\begin{array}{l}\text { [3059] bod yig gsal byed nyer dgu pa 'di'i nga ro ‘don tshul la skye gnas 'grin pa dang/ byed pa mgrin pa/ nang gi rtsol ba } \\
\text { mgrin pa phye ba dang/ phyi'i rtsol ba srog chen dro zhing sgra ldan pa/ ... 1.ming gzhi'i mo gsham gyi yi ge zhig ... 2.dbugs } \\
\text { kyi ha/ ... ha rgyag pa/ ... me long la ha 'debs pa/ ... }\end{array}$ \\
\hline & JV & number 29, alas, be in ecstasy, breath, yonder, further, picture, painting, collar of a coat \\
\hline & IW & a yawn, [moist] breath, HA, [laugh or letter], mo gsham gyi yi ge zhig \\
\hline & RY & a yawn, breath \\
\hline
\end{tabular}

I think they are right: Roger Bacon wrote it. He wrote it in the Tibetan Alphabet to hide the science from the church. Ah, Mother Church. Bacon had the instruments to see bacteria, he had an interest in medicine and science, and astrology. How did he get access to Egyptian Secrete? Through Aristotle who had access to Egyptian secrets, the secret of secrets. Whoever wrote the Voynich Manuscript (Tables 2 \& 3), had access to three things: firstly, Instruments such as a microscope, secondly knowledge of the Ancient Egyptian Mathematical "secrets"; and thirdly, knowledge of the Tibetan Alphabet [3]. I might decipher the manuscript. I purchased a copy of the book on Kindle. It is too small to read. You can pick up my collected works at LULU. com. They will be valuable in years to come as I plan to let only a few copies go for sale.

Table 2: kiss goddess dm, the invisible entity, one of a pairs.

\begin{tabular}{|c|c|c|}
\hline $4 \cdot$ & & [not found] \\
\hline 近 & IW,RY & a kiss \\
\hline \multirow{8}{*}{ के } & $\mathrm{JH}-\mathrm{NG}$ & food; to eat \\
\hline & $\mathrm{JH}-\mathrm{T}$ & bza'/ za/ bzas/ zos/ \\
\hline & OT & $\begin{array}{c}\text { [2441] bod yig gsal byed nyer gnyis pa 'di'i nga ro 'don tshul la skye gnas so dang/ byed pa lce rtse/ nang gi rtsol ba mgrin pa } \\
\text { phye ba dang/ phyi'i rtsol ba srog chen dro zhing sgra med pa/ ming gzhi'i mo yig cig ... }\end{array}$ \\
\hline & DM & $\begin{array}{l}\text { as a part of women's names, immediately following the clan name, may also be spelled bza', or, more problematically, tsha or } \\
\text { btsa'. }\end{array}$ \\
\hline & IW & $\begin{array}{l}\text { ZA [bod yig gsal byed nyer gnyis pa 'di'i nga ro 'don tshul la skye gnas so dang, byed pa lce rtse, nang gi rtsol ba mgrin pa phye } \\
\text { ba dang, phyi'i rtsol ba srog chen dro zhing sgra med pa, ming gzhi'i mo yig cig] \{dbang-gsum- three profound empowerments } \\
\text { [Gd-mk]. ZA [R] }\end{array}$ \\
\hline & $\mathrm{RY}$ & accept, take! \\
\hline & JV, IW, RY & beer \\
\hline & $\mathrm{JV}$ & *, lynx, invisible entity \\
\hline w & JH-ENG & one of a pair; $\{\mathrm{T}\}$ an odd number \\
\hline
\end{tabular}




\section{Global Journal of Archaeology \& Anthropology}

\begin{tabular}{|c|c|}
\hline OT & 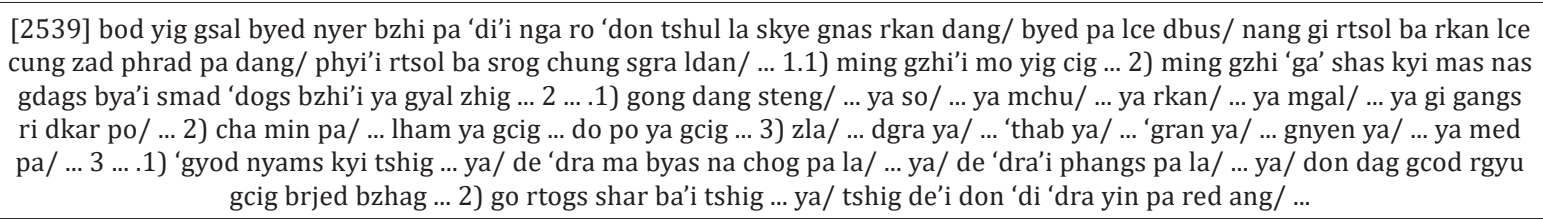 \\
\hline DM & $\begin{array}{l}\text { a sentence-final particle used with the imperative mood. Samdo A V 255r.1-6. Skal-ldan-rgya-mtsho uses it as a 'free' } \\
\text { interjection in post-verb position (the verb in this case is not in imperative mood). Sujata, Dissert. }\end{array}$ \\
\hline JV & smallness, littleness, equal, above, up odd \\
\hline IW & $\begin{array}{l}\text { 1) match, equal, rival; 2) up [wards] above, upper, higher; 3) after a verb: infinitive or 'for'; 4) YA; 5) cha min pa; 3) opponent; } \\
\text { 6) expression of regret; 7) } 0 \text { yes, I see; 7) 24; 8) smallness; 9) } 1 \text { of a pair }\end{array}$ \\
\hline RY & 1) match, equal. 2) up. 3) used after a verb to form the infinitive and 'for'; equal \\
\hline
\end{tabular}

Table 3: Kiss Goddess Dm, The Invisible Entity.

\begin{tabular}{|c|c|c|}
\hline 就 & IW, RY & a kiss \\
\hline \multirow[t]{6}{*}{$\nexists^{\prime}$} & JH-ENG & food; to eat \\
\hline & $\mathrm{JH}-\mathrm{T}$ & bza'/ za/ bzas/ zos/ \\
\hline & OT & $\begin{array}{l}\text { [2441] bod yig gsal byed nyer gnyis pa 'di'i nga ro 'don tshul la skye gnas so dang/ byed pa lce rtse/ } \\
\text { nang gi rtsol ba mgrin pa phye ba dang/ phyi'i rtsol ba srog chen dro zhing sgra med pa/ ming gzhi'i } \\
\text { mo yig cig... }\end{array}$ \\
\hline & $\mathrm{DM}$ & $\begin{array}{l}\text { as a part of women's names, immediately following the clan name, may also be spelled bza', or, more } \\
\text { problematically, tsha or btsa'. }\end{array}$ \\
\hline & IW & $\begin{array}{l}\text { ZA [bod yig gsal byed nyer gnyis pa 'di'i nga ro 'don tshul la skye gnas so dang, byed pa lce rtse, nang } \\
\text { gi rtsol ba mgrin pa phye ba dang, phyi'i rtsol ba srog chen dro zhing sgra med pa, ming gzhi'i mo yig } \\
\text { cig ] \{dbang-gsum- three profound empowerments [Gd-mk]. ZA [R] }\end{array}$ \\
\hline & RY & accept, take! \\
\hline \multirow[t]{2}{*}{ हो } & JV, IW, RY & beer \\
\hline & JV & *, lynx, invisible entity \\
\hline s. & OT & $\begin{array}{l}\text { [722] bod yig gsal byed lnga pa 'di'i nga ro 'don tshul la skye gnas rkan dang/ byed pa lce dbus/ nang } \\
\text { gi rtsol ba rkan lcer phrad pa dang/ phyi'i rtsol ba srog chung sgra med/ ... 1) ming gzhi'i pho yig cig ... } \\
\text { 2) ming mtha' la 'jug tshe skabs 'gar gsal byed cha dang 'dra ba/ ... cha ... brag ca/ ... lag ca/ ... mtshon } \\
\text { ca/ ... rgyan ca/ ... }\end{array}$ \\
\hline \multirow[t]{4}{*}{5} & JV, IW, RY & excrement \\
\hline & $\mathrm{JV}$ & number $5, *$, alvine discharges, SA lca \\
\hline & IW & CA. 1) ca; 2) male/ noun particle \\
\hline & RY & *; $\{$ ca 'dor ba $\}$ to discharge * \\
\hline \multirow[t]{8}{*}{ ब } & OT & $\begin{array}{l}\text { [1021] bod yig gsal byed dgu pa 'di'i nga ro 'don tshul la skye gnas so dang/ byed pa lce rtse/ nang gi } \\
\text { rtsol ba so dang lce rtse phrad pa dang/ phyi'i rtsol ba srog chung sgra med/ ming gzhi'i pho yig cig ... }\end{array}$ \\
\hline & $\mathrm{DM}$ & See 'tshod. \\
\hline & JV & door of admission to all things, figure 9 \\
\hline & IW & $\begin{array}{l}\text { TA [bod yig gsal byed dgu pa 'di'i nga ro 'don tshul la skye gnas so dang, byed pa lce rtze, nang gi rtzol } \\
\text { ba so dang lce rtze phrad pa dang, phyi'i rtzol ba srog chung sgra med, ming gzhi'i pho yig cig] }\end{array}$ \\
\hline & JH-ENG & mouth; opening; face; area; verb + kha or ka = about to (do something) \\
\hline & $\mathrm{JH}-\mathrm{OE}$ & \{C\}face; door; marvellous power(?); so also(?) \\
\hline & JH-SKT, YOGA & mukha \\
\hline & JH-SKT & $\{\mathrm{C}, \mathrm{MSA}\}^{*} ;\{\mathrm{C}\}$ razmi- ${ }^{*} ;\{\mathrm{C}\}$ daMzana \\
\hline
\end{tabular}




\begin{tabular}{|c|c|}
\hline OT & $\begin{array}{l}\text { [185] bod yig gsal byed gnyis pa 'di'i nga ro 'don tshul la skye gnas mgrin pa dang/ byed pa mgrin pa/ } \\
\text { nang gi rtsol ba mgrin pa phye ba dang/ phyi'i rtsol ba srog chen sgra med/ ... 1. 1) ming gzhi'i ma } \\
\text { ning yi ge zhig ... 2) ming sngon gyi cha shas shig ... kha dog ... kha 'thor ba/ ... kha sgyur ba/ ... 3) rtags } \\
\text { mtshungs 'dren skabs rjes 'jug ma ning gis drangs pa'i ming mtha' zhig ... dgun kha/ ... gter kha/ ... gsol } \\
\text { kha/ ... } 2 \text {... 1) skye 'gro'i zas za ba dang smra ba'i sgo/ ... kha lkugs pa/ ... bong bu'i kha nas gser bskyug } \\
\text { mi yong/ ... ming gi rnam grangs la ngag 'don dang/ gtam gyi 'byung gnas/ bdud rtsi'i rten/ mu kha/ } \\
\text { smra ba'i sgo/ smra byed/ zhal/ za byed/ gsung byed bcas so/ 2) snod kyi kha/ ... phor ba'i kha/ ... } \\
\text { bum pa'i kha/ ... shel dam gyi kha gcod pa/ ... 3) mdun phyogs/ ... khang pa phan tshun kha sprod pa/ } \\
\text {... kha phyogs/ ... 4) nye 'gram/ ... mtsho kha/ ... gtsang po'i kha/ ... 5) steng cha/ ... khri kha/ ... dpe cha } \\
\text { cog tse'i kha la bting ba/ ... 6) dus skabs/ ... phyogs la 'gro kha/ ... las ka byed khar bsam blo gzab gzab } \\
\text { gtong dgos/ ... 7) skad cha/ ... rdzun shod mkhan gyi kha la mnyan rgyu med/ ... gros bsdur byed mi } \\
\text { tshang ma kha mthun pa/ ... 8) phyugs kyi lo tshad/ ... g.yag kha rgan/ ... rta kha chung/ ... 9) ras kyi 'jal } \\
\text { tshad zheng dkyus 'dra ba zhig ... ras kha gang/ ... gos chen kha do/ ... }\end{array}$ \\
\hline YOGA & Asya \\
\hline $\mathrm{DM}$ & $\begin{array}{l}\text { = kha sang kha'i nyi ma. 'der gestrige Tag' Kaschewsky 82. unmittelbar bevor. Kaschewsky2. a 'secret' } \\
\text { way of saying 'five,' used by gamblers. Norbu, Drung 229, n. 65.. 'mouth.' DD illus. } 17 .\end{array}$ \\
\hline JV & $\begin{array}{l}\text { bitter, second, part, time, front side, face, mouth, surface, square of cloth, second, } 2 \text {, optional additional } \\
\text { syllable to many colloquial words, part, origin, source, time, breadth of cloth, language, conversation, } \\
\text { word, } 1 / 6 \text { part of a tang ka coin, mouth, edge, direction, to befall, to involuntarily happen, five }\end{array}$ \\
\hline IW & $\begin{array}{l}\text { KHA ; 1) neuter particle; 2) prefix cha shas shig...Kha dog, Kha 'thor ba, Kha sgyur ba,...3) Rtags } \\
\text { mtsungs 'dren skabs neuter suffix: Dgun kha,...Gter kha,...Gsol kha,...2...1) mouth [syn: ngag 'don dang, } \\
\text { gtam gyi 'byung gnas, bdud rtsi'i rten, mu kha, smra ba'i sgo, smra byed, zhal, za byed, gsung byed] } \\
\text { 2) mouth of a vessel; 3) front side; 4) shore, bank; 5) top surface [Khri kha, cog tse'i kha] (6) time, } \\
\text { occasion ['gro kha,...Las ka byed kha] (7) talk, what is said (8) age of an animal [kha rgan ching] (9) } \\
\text { measure of a piece of cloth [Ras kha gang,...Goschen kha do]. 1) face, front; 2) lips, mouth, snout; 3) } \\
\text { words, talk, say; 4) opening, bank, shore, rim, surface; 5) measurement = } 1 \text { square [of width given]; } \\
\text { 6) direction; 7) appearance; 8) the letter kha used in alphabetical ordering; 9) guardian, adjutant, } \\
\text { body-guard; 10) experience physically/by sense, smack, interfere, meddle with; 11) promise; 12) kiss, } \\
\text { inveigh; 13) part; 14) snow; 15) at the verge of, about to; 16) bitter; 17) some, several. KHA, [2nd in } \\
\text { alphabetical order]; 1) neuter particle; 2) prefix cha shas shig; 3) Rtags mtshungs 'dren skabs neuter } \\
\text { suffix; 4) mouth * [of a vessel]; 5) front [side]; 6) shore, bank; 7) top surface [Khri kha, cog tse'i kha]; } \\
\text { 8) time, occasion ['gro kha,...Las ka byed kha]; 9) words, talk, say, what is said; 10) age of an animal; } \\
\text { 11) measure of a piece of cloth* face, lips, cover, snout, opening, rim, surface, a measurement equaling } \\
\text { 1 square of whatever the width is, direction, appearance, guardian, adjutant, body-guard, experience } \\
\text { physically, smack, interfere, meddle with, promise, kiss, inveigh, part, 1st particle in many words, } \\
\text { snow, part, at the verge of, about to, bitter, some, several }\end{array}$ \\
\hline $\mathrm{RB}$ & mouth; face \\
\hline RY & $\begin{array}{l}\text { mottled. + lta - facing. maw. countenance; (kha bkrag yal le ba\} a beautiful, bright countenance. } \\
\text { words, speech/ in the middle; the direction one is facing; 1) face, front, lips, mouth, cover, snout. 2) } \\
\text { words, talk, say. 3) opening. 4) bank, shore. 5) rim, surface. 6) a measurement equaling one square of } \\
\text { whatever the width is. 7) direction. 8) appearance. 9) the letter kha used in alphabetical ordering. 10) } \\
\text { vi. to experience physically. 11) first particle in many word combinations. 12) at the verge of, about } \\
\text { to. 13) bitter, snow, part, some, several, to smack, to interfere, to meddle with, to promise, to kiss, to } \\
\text { inveigh. 14) guardian, adjutant, body-guard; summit/ at the time of, point of }\end{array}$ \\
\hline
\end{tabular}




\section{Global Journal of Archaeology \& Anthropology}

So, I've taken 243 credit hours $=81$ courses $1 / 81=0.012345679$ In french, 81 is quatre vignt et une 4 X 20 $+1=81421$ That is TIBIT If you consider that a 3 -credit hour course is 3402 hours of lecture plus another 3402 hours of home study, that is 6804 hours of study. $1 / 6.804=0.1469=1-$ $0.853=1-$ Sin $1=1-\operatorname{Cos} 1$ So 6804 hours of study is what is need at a minimum to understand the universe. I've studied for 48 years. The two pole problem is knowledge vs ignorance. I'll let this blog go until it hits 50,000 viewers. I'm told by an eminent Harvard scholar that these sorts of things must be checked and verified by the scholarly community. He's right. I hope some takes an interest in every sphere that I've deviled into and finds all my mistakes and corrects them. I can't get published or even reviewed for a scholarly Journal. I've sent dozens of papers to 16 professors at the local universities without one comment back. Apparently, they are not interested. Neither am I. I'm not getting paid. They are! I've tried more than a dozen universities around the world. All I got back were my expensive books. University is big business. their customers are unwitting young people. They really are not interested in cutting edge research. They are too jealous. That's what I;'ve learned after 48 years of study.

This blog has a life of its own. We're up to 519 readers today already. Enjoy! I'm going to bury myself in mathematica (Figure 10) (Tables 4 \& 5).

Table 4: Thaga Zatsha Distance Food.

\begin{tabular}{|c|c|c|}
\hline \multirow{6}{*}{ 子 } & OT & $\begin{array}{l}\text { [722] bod yig gsal byed lnga pa 'di'i nga ro 'don tshul la skye gnas rkan dang/ byed pa lce dbus/ nang gi rtsol ba rkan } \\
\text { lcer phrad pa dang/ phyi'i rtsol ba srog chung sgra med/ ... 1) ming gzhi'i pho yig cig ... 2) ming mtha' la 'jug tshe } \\
\text { skabs 'gar gsal byed cha dang 'dra ba/ ... cha ... brag ca/ ... lag ca/ ... mtshon ca/ ... rgyan ca/ ... }\end{array}$ \\
\hline & JV, IW, RY & excrement \\
\hline & $\mathrm{JV}$ & number 5 , *, alvine discharges, SA lca \\
\hline & IW & CA. 1) ca; 2) male/ noun particle \\
\hline & RY & *; $\{$ ca 'dor ba\} to discharge * \\
\hline & OT & $\begin{array}{l}\text { [1021] bod yig gsal byed dgu pa 'di'i nga ro 'don tshul la skye gnas so dang/ byed pa lce rtse/ nang gi rtsol ba so dang } \\
\text { lce rtse phrad pa dang/ phyi'i rtsol ba srog chung sgra med/ ming gzhi'i pho yig cig ... }\end{array}$ \\
\hline \multirow{7}{*}{5} & DM & See 'tshod. \\
\hline & JV & door of admission to all things, figure 9 \\
\hline & IW & $\begin{array}{c}\text { TA [bod yig gsal byed dgu pa 'di'i nga ro 'don tshul la skye gnas so dang, byed pa lce rtze, nang gi rtzol ba so dang lce } \\
\text { rtze phrad pa dang, phyi'i rtzol ba srog chung sgra med, ming gzhi'i pho yig cig] }\end{array}$ \\
\hline & JH-ENG & mouth; opening; face; area; verb + kha or ka = about to (do something) \\
\hline & $\mathrm{JH}-\mathrm{OE}$ & $\{C\}$ face; door; marvellous power(?); so also(?) \\
\hline & JH-SKT, YOGA & mukha \\
\hline & JH-SKT & $\{\mathrm{C}, \mathrm{MSA}\}^{*} ;\{\mathrm{C}\}$ razmi-*; $\{\mathrm{C}\}$ daMzana \\
\hline \multirow[t]{3}{*}{ R } & OT & 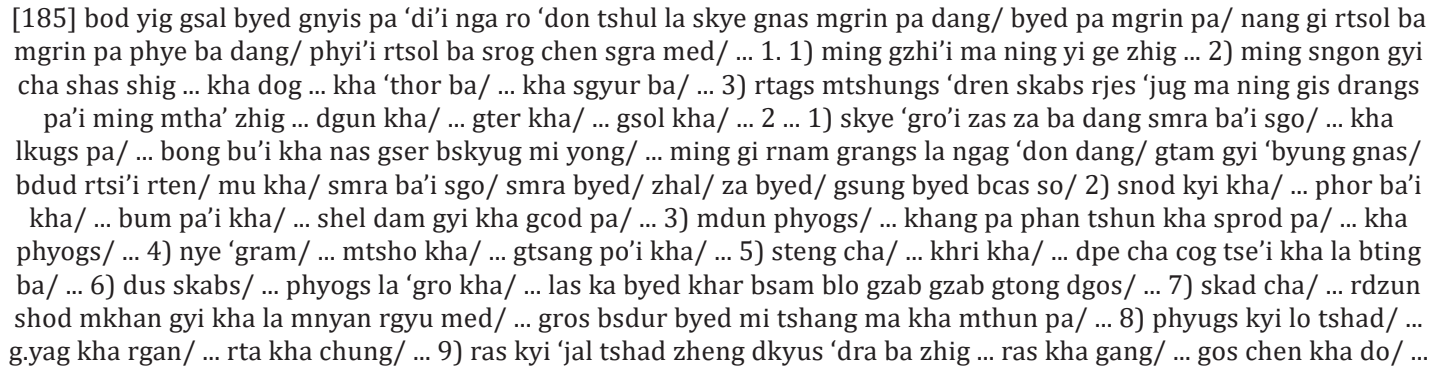 \\
\hline & YOGA & Asya \\
\hline & $\mathrm{DM}$ & $\begin{array}{l}\text { = kha sang kha'i nyi ma. 'der gestrige Tag' Kaschewsky } 82 \text {. unmittelbar bevor. Kaschewsky2. a 'secret' way of saying } \\
\text { 'five,' used by gamblers. Norbu, Drung 229, n. 65.. 'mouth.' DD illus. } 17 \text {. }\end{array}$ \\
\hline
\end{tabular}




\section{Global Journal of Archaeology \& Anthropology}

\begin{tabular}{|c|c|c|}
\hline & JV & $\begin{array}{l}\text { bitter, second, part, time, front side, face, mouth, surface, square of cloth, second, } 2 \text {, optional additional syllable to } \\
\text { many colloquial words, part, origin, source, time, breadth of cloth, language, conversation, word, } 1 / 6 \text { part of a tang ka } \\
\text { coin, mouth, edge, direction, to befall, to involuntarily happen, five }\end{array}$ \\
\hline \multirow[t]{3}{*}{ A } & IW & $\begin{array}{l}\text { KHA ; 1) neuter particle; 2) prefix cha shas shig...Kha dog, Kha 'thor ba, Kha sgyur ba,...3) Rtags mtsungs 'dren skabs } \\
\text { neuter suffix: Dgun kha,...Gter kha,...Gsol kha,...2...1) mouth [syn: ngag 'don dang, gtam gyi 'byung gnas, bdud rtsi'i } \\
\text { rten, mu kha, smra ba'i sgo, smra byed, zhal, za byed, gsung byed] 2) mouth of a vessel; 3) front side; 4) shore, bank; } \\
\text { 5) top surface [Khri kha, cog tse'i kha] (6) time, occasion ['gro kha,...Las ka byed kha] (7) talk, what is said (8) age } \\
\text { of an animal [kha rgan ching] (9) measure of a piece of cloth [Ras kha gang,...Goschen kha do]. 1) face, front; 2) lips, } \\
\text { mouth, snout; 3) words, talk, say; 4) opening, bank, shore, rim, surface; 5) measurement = 1 square [of width given]; } \\
\text { 6) direction; 7) appearance; 8) the letter kha used in alphabetical ordering; 9) guardian, adjutant, body-guard; 10) } \\
\text { experience physically/by sense, smack, interfere, meddle with; 11) promise; 12) kiss, inveigh; 13) part; 14) snow; 15) } \\
\text { at the verge of, about to; 16) bitter; 17) some, several. KHA, [2nd in alphabetical order]; 1) neuter particle; 2) prefix } \\
\text { cha shas shig; 3) Rtags mtshungs 'dren skabs neuter suffix; 4) mouth * [of a vessel]; 5) front [side]; 6) shore, bank; 7) } \\
\text { top surface [Khri kha, cog tse'i kha]; 8) time, occasion ['gro kha,...Las ka byed kha]; 9) words, talk, say, what is said; } \\
\text { 10) age of an animal; 11) measure of a piece of cloth * face, lips, cover, snout, opening, rim, surface, a measurement } \\
\text { equaling } 1 \text { square of whatever the width is, direction, appearance, guardian, adjutant, body-guard, experience } \\
\text { physically, smack, interfere, meddle with, promise, kiss, inveigh, part, 1st particle in many words, snow, part, at the } \\
\text { verge of, about to, bitter, some, several }\end{array}$ \\
\hline & $\mathrm{RB}$ & mouth; face \\
\hline & RY & $\begin{array}{l}\text { mottled. + lta - facing. maw. countenance; \{kha bkrag yal le ba\} a beautiful, bright countenance. words, speech/ in the } \\
\text { middle; the direction one is facing; 1) face, front, lips, mouth, cover, snout. 2) words, talk, say. 3) opening. 4) bank, } \\
\text { shore. 5) rim, surface. 6) a measurement equaling one square of whatever the width is. 7) direction. 8) appearance. } \\
\text { 9) the letter kha used in alphabetical ordering. 10) vi. to experience physically. 11) first particle in many word } \\
\text { combinations. 12) at the verge of, about to. 13) bitter, snow, part, some, several, to smack, to interfere, to meddle } \\
\text { with, to promise, to kiss, to inveigh. 14) guardian, adjutant, body-guard; summit/ at the time of, point of }\end{array}$ \\
\hline
\end{tabular}

Table 5: TIB-BIT.

\begin{tabular}{|c|c|}
\hline $\begin{array}{l}\text { JH-ENG, JV,IW, } \\
\text { RY }\end{array}$ & distance \\
\hline OT & [1136] sa cha gnyis bar gyi ring tshad dam bar khyon/ ... sa thag le dbar khri phrag gis chod pa/ ... \\
\hline DM & as used to form comparatives or superlatives, see under chung thag (BBNP 469). \\
\hline $\mathrm{JV}$ & place in tibet, palate, ${ }^{*}$, web \\
\hline IW & 1) ${ }^{*}$, distant, a great *; 2) [vb+ma+--] as soon as; 3) texture, weave, web \\
\hline RY & $\left.{ }^{*} ; 1\right)^{*}$, distant, a great *. 2) [vb+ma+] as soon as 3) sometimes used for $\{$ thags $\}$ \\
\hline JH-ENG & food; to eat \\
\hline $\mathrm{JH}-\mathrm{T}$ & bza'/ za/ bzas/ zos/ \\
\hline OT & $\begin{array}{l}\text { [2441] bod yig gsal byed nyer gnyis pa 'di'i nga ro 'don tshul la skye gnas so dang/ byed pa Ice rtse/ nang gi } \\
\text { rtsol ba mgrin pa phye ba dang/ phyi'i rtsol ba srog chen dro zhing sgra med pa/ ming gzhi'i mo yig cig ... }\end{array}$ \\
\hline DM & $\begin{array}{c}\text { as a part of women's names, immediately following the clan name, may also be spelled bza', or, more } \\
\text { problematically, tsha or btsa'. }\end{array}$ \\
\hline IW & $\begin{array}{c}\text { ZA [bod yig gsal byed nyer gnyis pa 'di'i nga ro 'don tshul la skye gnas so dang, byed pa lce rtse, nang gi rtsol } \\
\text { ba mgrin pa phye ba dang, phyi'i rtsol ba srog chen dro zhing sgra med pa, ming gzhi'i mo yig cig ] \{dbang- } \\
\text { gsum- three profound empowerments [Gd-mk]. ZA [R] }\end{array}$ \\
\hline
\end{tabular}

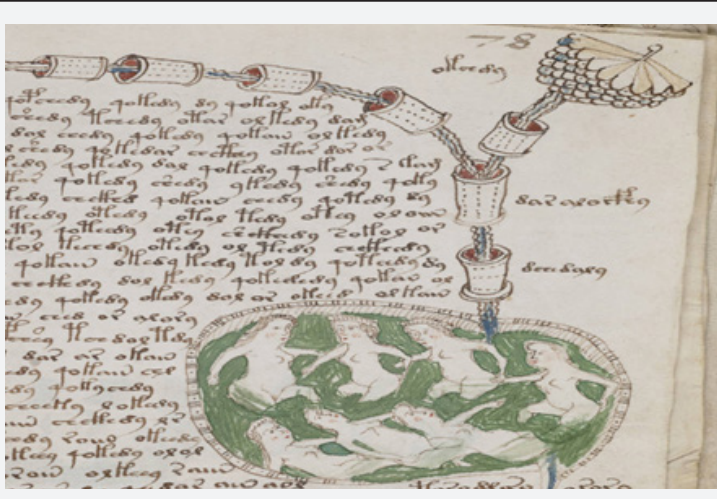

Figure 10: Nyphna Goddesses Ca Ta Kha About to Discharge Something from Your Mouth.
TIB-BIT

$400+10+22+10+400$

$=421+421$

$=842 \sim 0.8415$

$=\operatorname{Sin} 1=\operatorname{Cos} 1$

Human knowledge is converging rapidly now thanks to the transistor, the computer, internet, and Wikipedia. Western civilization is arrogant. We live in an abstract world that disregards nature. "Tis a sick bird that shits in its own nest." Humans had more knowledge with far less technology that I mentioned. It went slower, but they had figured out more than 
our scientists did. The West has been at it for 2000 years. The Buddhist have been at it for 7000 . The Hebrews have been at it for 6000 . The Egyptians have been at it for 4500 years. I've been at it for 47 years, but I was schooled in western thought for 18 years. Now it comes to an end at just over 3000 posts. I wonder how many days we have remaining? 10? We're close to the end anyhow. The Jews taught us all. did they get it from the Egyptians or the Babylonians? All of humanity is connected. Let's live in peace (Figures 11-15).

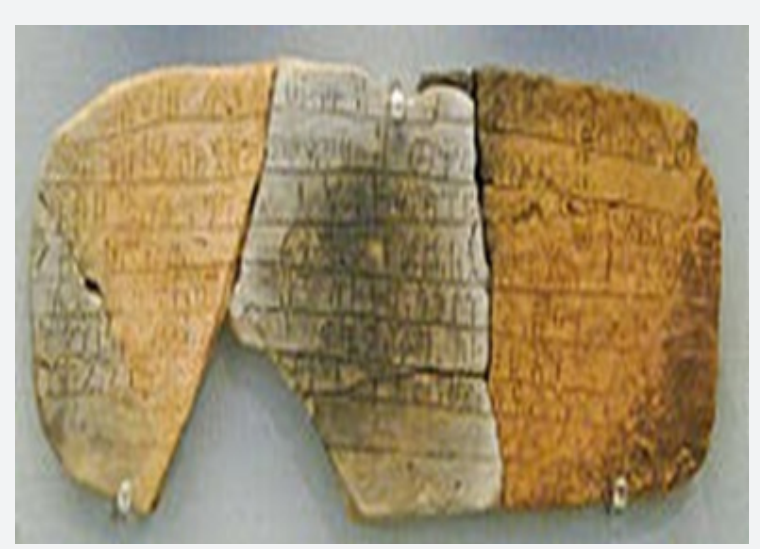

Figure 11: Greek Linear B. I decoded this as a Hebrew tablet.

\begin{tabular}{|c|c|c|c|c|c|c|c|}
\hline$\pi$ & ka [ká] & K & kha [kª́] & पा & ga $\left[k^{2} / k^{\text {hà }}\right]$ & $\Sigma^{*}$ & ña [gà] \\
\hline 5 & ca [tyá] & $\Phi^{\circ}$ & cha [tyád & $E^{\cdot}$ & ja [yà $\}$ & 3 & ña [ nà̀] \\
\hline 5 & ta [tá] & घ* & tha [सá] & 5 & da [tà/thà] & $\overline{9}$ & na [nà] \\
\hline $\mathrm{Cr}^{\circ}$ & pa [pá] & 땅 & pha [płá] & $5^{*}$ & ba [pà/płà] & 추 & ma [mà] \\
\hline రా & tsa [tsá] & ळ゙ & tsha [ts`áa] & $E^{*}$ & $\mathrm{dza}$ [tsà] & & \\
\hline $\mathbb{3}^{*}$ & wa [wà] & व. & ža [jà] & $\Xi^{*}$ & za [sà] & & \\
\hline$R^{\circ}$ & ('a) [à] & w & ya [jà] & $\Sigma \cdot$ & ra [rà] & $2 r$ & la [là] \\
\hline ש. & & 9 & ša [ Já] & $\mathrm{N}^{\circ}$ & sa [sá] & & \\
\hline 7 & ha [há] & ov & (a) [á] & & & & \\
\hline
\end{tabular}

Figure 12: Babylonians.

\begin{tabular}{|c|c|c|c|c|c|c|c|}
\hline \multicolumn{8}{|c|}{ Tibetan Consonants } \\
\hline गा & 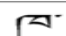 & वा & $=-$ & उ- & ๘ & $E$ & s. \\
\hline ka & $k^{\prime} \mathbf{a}$ & $\mathrm{g}^{2} \mathrm{a}$ & nga & cha & ch'a & j-a & nya \\
\hline 5 & $\nabla^{\circ}$ & 5 & क & $\approx r$ & $\approx$ & 口י & वr \\
\hline ta & $\mathbf{t}^{2} \mathbf{a}$ & $d^{\prime} a$ & na & pa & $p^{-a}$ & b'a & $\mathrm{ma}$ \\
\hline 5 & क- & $E$ & 世 & ब & $\nexists^{-}$ & $\approx$ & ख्य \\
\hline tsa & ts'a & $d z^{\prime} a$ & wa & zha & $z a$ & a & ya \\
\hline 工́ & r & -9 & $\approx \mathbf{V}^{-}$ & ラ & $\mathbb{V}^{\circ}$ & & \\
\hline ra & $1 \mathrm{a}$ & sha & sa & ha & a & & \\
\hline \multicolumn{8}{|c|}{ Tibetan Vowel Modifiers } \\
\hline \multicolumn{2}{|c|}{ खें } & \multicolumn{2}{|c|}{ खु } & \multicolumn{2}{|c|}{ खें } & \multicolumn{2}{|c|}{ जे } \\
\hline \multicolumn{2}{|c|}{ i } & \multicolumn{2}{|c|}{$\mathbf{u}$} & \multicolumn{2}{|c|}{0} & \multicolumn{2}{|c|}{0} \\
\hline
\end{tabular}

Figure 13: Tibetan Alphabet.Look in the manuscript for the goddess $\mathrm{IO}$ and $\mathrm{ECHO}$ above.

\section{$5,20,8,70$ \\ $8,7,5,2$ \\ $\mathrm{ECHO}$ \\ eta, ka,ha,o}

Figure 14: Tibetan Alphabets

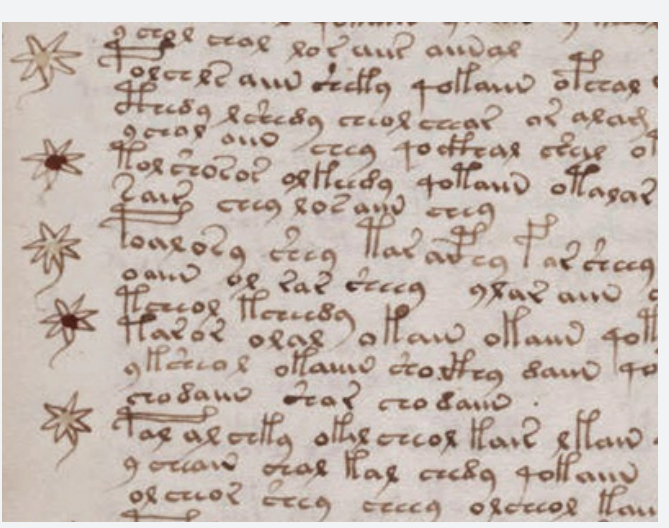

Figure 15: Natural Hallucinogens to Determine that the Human Mind.

Greek Linear B. I decoded this as a Hebrew tablet. The Tibetan Alphabet comes from the Hebrew alphabet I think. The Hebrews come from the Egyptian. As the Hebrews spread around the world, they took Egyptian knowledge about the universe with them. Should be exciting to read the Nymph book. Its the Tibetan Bible. The Jewish Tribe of Manasseh floated through Tibet or china on their way to North America. Manasseh got their knowledge from Moses who was educated by the Egyptians. All this cosmology spring from the Egyptians (Babylonians?) who recorded it in their pyramids. So, did the Mayans (Figure 16).

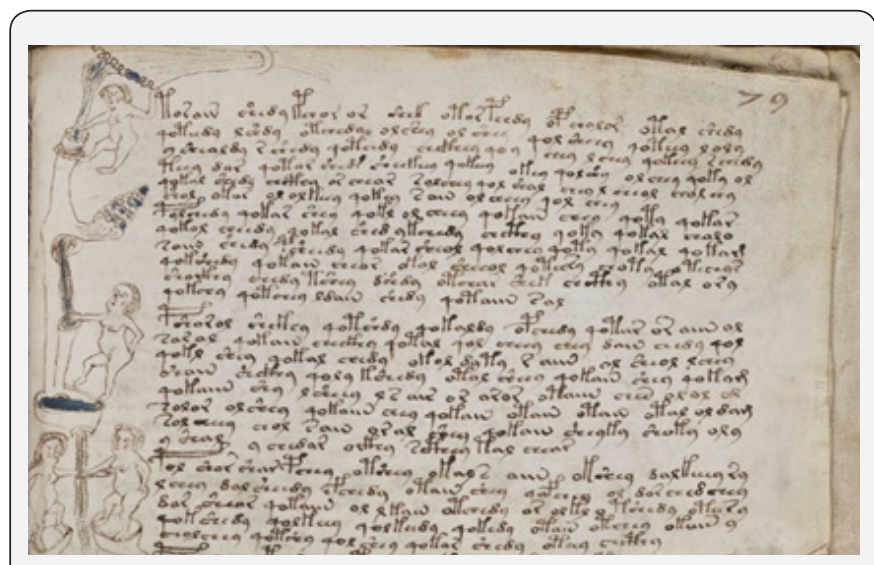

Figure 16: Bit Greek Mythology. 
Dec 21, 2012+4 BCE-1 (No year zero) =Dec 21, 2015But the Hebrews count the first day as 1 year. So, subtract another year. I leave it to you to play with these numbers. Tibetan Alphabet. Look in the manuscript for the goddess IO and ECHO above. So, if you use Tibetan Alphabets, you van read about Mother Nature and the peak of the Tibetan Culture. They must have used natural hallucinogens to determine that the human mind functioned at $31.8 \sim 32 \mathrm{~Hz}$.

\section{Conclusion}

What the Voynic Manuscript reveals is that the culture of the world is intertwined. From the Egyptians in Africa, to the UK on Europe, to Khazachstan in Cenbtral Asia, to Tibet, to the
Mayans in the Americans, the same religious knowledge has been transferred to all the peoples of the world thanks largely to the Israelites. Philosophically, God is available to everyone. The Voynich Manuscript is just one piece of that large puzzle of human knowledge.

\section{References}

1. Images-Voynich Manuscript.

2. The voynich manuscript the mysterious code vthat has defied intrepretation for centuries, g. Kennedy and r. Churchill, inner traditions rocheser vermont.

3. Voynich Manuscript, Yale University, USA.

\section{Your next submission with Juniper Publishers} will reach you the below assets

- Quality Editorial service

- Swift Peer Review

- Reprints availability

- E-prints Service

- Manuscript Podcast for convenient understanding

- Global attainment for your research

- Manuscript accessibility in different formats

( Pdf, E-pub, Full Text, Audio)

- Unceasing customer service

Track the below URL for one-step submission https://juniperpublishers.com/online-submission.php 